\title{
L'Affaire Louvel ou l'introuvable complot. Evénement, enquête judiciaire et expression politique dans la France de la Restauration.
} Thèse de doctorat d'histoire soutenue le 14 décembre 2005 à l'université Paris-XII-Val-de-Marne, devant un jury composé de Florence Bourillon (présidente), Jacques-Olivier Boudon, Philippe Boutry (directeur de la thèse), Dominique Kalifa, Natalie Petiteau.

\section{Gilles Malandain}

\section{OpenEdition} Journals

Édition électronique

URL : http://journals.openedition.org/rh19/1081

DOl : $10.4000 /$ rh 19.1081

ISSN : $1777-5329$

Éditeur

La Société de 1848

Édition imprimée

Date de publication : 1 décembre 2005

ISSN : 1265-1354

\section{Référence électronique}

Gilles Malandain, «L'Affaire Louvel ou l'introuvable complot. Evénement, enquête judiciaire et expression politique dans la France de la Restauration. », Revue d'histoire du XIXe siècle [En ligne], 31 2005, mis en ligne le 27 juin 2006, consulté le 22 septembre 2020. URL : http:// journals.openedition.org/rh19/1081; DOI : https://doi.org/10.4000/rh19.1081

Ce document a été généré automatiquement le 22 septembre 2020.

Tous droits réservés 


\section{L'Affaire Louvel ou l'introuvable complot. Evénement, enquête judiciaire et expression politique dans la France de la Restauration.}

Thèse de doctorat d'histoire soutenue le 14 décembre 2005 à l'université Paris-XII-Val-de-Marne, devant un jury composé de Florence Bourillon (présidente), Jacques-Olivier Boudon, Philippe Boutry (directeur de la thèse), Dominique Kalifa, Natalie Petiteau.

\section{Gilles Malandain}

1 Cette thèse est le résultat de plusieurs années de travail sur l'assassinat du duc de Berry en $1820^{1}$. Le sujet a d'abord été défini, au stade du DEA, comme un point d'entrée ou un observatoire sur les cultures politiques de l'époque post-révolutionnaire. Mon intérêt initial ne portait pas tant sur l'événement, a priori connu, que sur l'enquête consécutive de la Cour des pairs chargée du procès de Louvel ${ }^{2}$. A partir de ce gisement d'archives judiciaires et policières, encore jamais exploré, je comptais d'abord m'interroger d'une part sur les formes concrètes et quotidiennes de l'opinion publique, notamment au plus bas de l'échelle sociale, et d'autre part sur les moyens de leur saisie et de leur réinsertion dans le discours historique. Tout en ayant à cœur l'horizon problématique que constitue la crise actuelle de la démocratie, il s'agissait de prendre la question très en amont, avant l'âge du vote et celui des sondages, lorsque cette "opinion " paraît encore relativement improbable. Loin de se donner en effet comme une évidence, l'expression populaire, tout en fondant de droit la démocratie contemporaine, en constitue aussi un repoussoir, tant elle est réputée, notamment chez ceux qui dirigent le débat politique, irrationnelle, incompétente, ingérable. Hier comme aujourd'hui, elle suscite une tension et des tentations contradictoires chez les gouvernants : tentation du mépris et du refoulement d'une part, scrutation inquiète et tentation pédagogique ou « communicante » de l'autre. 
2 Nul doute que l'origine de cette tension ne remonte au XVIII ${ }^{e}$ siècle, au moment de la formation d'un espace public libéral et de la crise de l'absolutisme. Mais les enjeux du siècle des Lumières se rejouent de manière plus cruciale encore dans les décennies qui suivent la grande tourmente révolutionnaire, lorsqu'il faut répondre à la " nécessité de l'assemblage » qu'évoque Alain Corbin en parlant de «l'invention du XIX siècle ». La démonstration de l'intérêt génétique de l'âge censitaire n'est du reste plus à faire : la Restauration et l'Empire, plus tardivement que la Monarchie de Juillet, sont pleinement rentrés aujourd'hui dans le champ historiographique. A mi-chemin entre 1789 et 1848, au cœur d'un premier tiers du XIX siècle particulièrement avare de politique populaire, l'affaire Louvel offrait une prise originale et précieuse. Grâce surtout à l'importance prise par l'enquête judiciaire, elle permettait de croiser des sources diverses et en même temps des niveaux très variés d'expérience, de discours ou de pratique, réunis dans une même conjoncture, et autour d'elle.

3 Il fallait cependant prendre le parti un peu risqué de limiter l'horizon diachronique, nécessaire à tout travail d'histoire, pour tenter l'analyse intensive d'un corpus ponctuellement situé dans la chronologie. Toutefois, il existait des modèles convaincants, qui avaient montré la valeur d'une telle démarche : ainsi de riches études portant sur de «grands » attentats politiques, comme celui de Damiens ou l'assassinat de Marat; ou encore des travaux ayant pris pour objet une affaire judiciaire, comme l'affaire Fualdès ou surtout l'affaire Lacenaire (A.-E. Demartini). Entre Damiens et Lacenaire, l'«affaire Louvel», pouvait se définir à la fois comme séquence événementielle ouverte par l'attentat régicide et comme dossier judiciaire. Mais il s'agissait d'autant moins d'imiter ce qui avait déjà été fait, que le sujet différait, au fond, des points de référence que je viens d'évoquer. D'un côté, l'essentiel, au-delà de l'événement lui-même, était de réintégrer pleinement l'enquête et ses résultats dans l'histoire politique de la Restauration; de l'autre, l'affaire n'était pas aussi nettement focalisée sur un acteur principal ni aussi nettement constituée comme telle, au plan judiciaire ou médiatique, que dans le cas de Lacenaire. J'ai néanmoins adopté volontiers cette notion d'affaire, pour sa valeur unifiante; mais il faut bien reconnaître le caractère hétérogène et problématique de l'unité que le terme suggère. Autrement dit, ni l'assassinat du duc de Berry en lui-même, ni la seule personnalité de Louvel, quel que soit l'intérêt qu'on peut leur porter, ne pouvaient suffire à définir l'affaire dans sa globalité comme un objet d'histoire.

4 Il fallait donc préciser ce que pouvait être le dénominateur commun à la polémique qui traverse l'espace public censitaire au lendemain de l'assassinat, aux propos tenus par l'assassin dans sa cellule, et à l'effervescence rumorale que les sources judiciaires permettaient de percevoir. Il fallait également s'efforcer d'intégrer les différents niveaux de discours rencontrés dans les diverses sources, sans tomber dans la naïveté d'une mise à plat globale et indifférenciée de phénomènes aussi radicalement dissemblables qu'un article du Conservateur et une conversation de cabaret. Tenter de restituer la polyphonie des réponses à l'événement, et en particulier celles qui ont laissé les traces les plus ténues, ne doit pas, en effet, conduire à négliger ou à minorer le poids contraignant des structures sociales, qui ne donnent pas à toutes les voix le même statut, la même audibilité.

5 J'ai donc cherché non à mélanger mais à articuler les différents niveaux de perception et d'usage de l'événement. En partant de ceux qui s'offrent le plus immédiatement - à travers la production imprimée notamment, ou par les canaux institutionnels - et en 
allant vers ceux que seules les sources de la surveillance ou de la répression permettent de repérer. Entre les deux pôles, l'enquête s'imposait décidément comme le cœur et le pivot de la recherche. C'est par l'enquête, en effet, qu'on pouvait réellement confronter le ténor politique, dénonciateur occasionnel ou objet de surveillance, et l'humble paysan, signalé comme témoin de propos suspects ou accusé d'avoir annoncé la mort du prince. Dans son principe comme dans sa pratique, l'enquête fait la jonction entre le discours politique légitime et le "bruit " populaire; processus complexe, collectif et " interactif», elle confronte le pays légal au pays réel, la partie significativement émergée de l'iceberg social et politique à sa profondeur présente-absente.

6 Cette enquête recherchait le complot dont Louvel était supposé avoir été l'exécutant ou "l'instrument »; mais ne le trouvant pas, elle se prolonge presque indéfiniment faute de pouvoir circonscrire sa cible. Son indétermination spécifique lui confère une ampleur, une "ouverture " géographique ou sociologique, et au total une dimension informative exceptionnelles. Avec l'enquête judiciaire, c'était donc la quête d'un complot introuvable, entre conspiration(s) possible(s) et conjuration fantasmatique, qui se trouvait placée au cœur du travail. La thèse y a trouvé non seulement un titre, mais je crois un gage d'unité pratique et théorique.

7 A l'intersection de l'action politique, du soupçon judiciaire et de l'imaginaire social, le complot, dénoncé, recherché, imaginé, aussi obsédant qu'évanescent, constitue bien, au bout du compte, ce «dénominateur commun » de l'affaire, qui ne devait pas, pour le coup, rester introuvable. Omniprésent dans les esprits, quoique sous des formes variées et parfois contradictoires, le complot est finalement la figure la plus consensuelle des discours ou des représentations politiques de 1820, même si ce consensus peut se faire dans l'exclusion réciproque. C'est du complot que parlent à la fois, dans des cadres et en des termes éventuellement différents, l'écrivain et sa portière, ou le propriétaire et l'artisan. Des disputes parlementaires jusqu'aux rumeurs parcourant les routes et les villages, c'était bien au fond la même interrogation sur les ressorts de l'événement, c'est-à-dire sur les ressorts de l'histoire contemporaine, trente ans après le début de la Révolution. C'était bien cette question qu'on me posait parfois : qui a véritablement tué le duc de Berry? Mais l'enjeu central de la recherche n'était pas tant d'y répondre positivement, bien sûr, que d'en montrer le rôle structurant dans la France de l'époque ; de montrer, autrement dit, que le complot, hypothèse récurrente et catégorie de perception, est l'une des clés possibles de la société du premier XIX ${ }^{e}$ siècle, une société hantée par son insupportable opacité à elle-même.

8 Pour mettre au jour les diverses manières dont le complot est appréhendé et utilisé dans la France de l'époque, la thèse s'organise donc en trois parties qui mettent successivement l'accent sur l'événement, pris surtout au sommet de la structure sociale et politique, sur l'enquête ensuite, et enfin sur l'expression politique populaire.

9 1) La richesse des renouvellements actuels du questionnaire des sciences historiques sur l'événement, comme sur les modes d'action politiques, mais aussi l'expérience que nous avons faite récemment d'attentats marquants et puissamment médiatisés - qui sollicitaient d'autant plus la réflexion que la problématique du terrorisme ou du fanatisme, et de l'usage de leur dénonciation, faisaient de façon évidente écho à l'affaire Louvel -, m'ont poussé à un retour plus long que prévu sur le 13 février et sur la crise que l'événement déclenche ou qu'il révèle.

10 Je ne pouvais pas réduire le début de la thèse à un récit introductif ou à une esquisse du décor de l'enquête. Pour éviter l'écueil d'une approche trop statique de l'événement - 
une approche qui ne le concevrait que comme "révélateur»de structures ou de représentations préexistantes -, et pour poser la question de l'action spécifique de l'événement dans le cours de l'histoire, je l'ai d'abord envisagé comme l'objet d'une expérience individuelle et collective. En s'appuyant sur les témoignages les plus accessibles, la thèse s'efforce de montrer concrètement ce que l'événement fait à la société qui l'éprouve, de quelle manière il «change la donne " en provoquant une brutale rupture dans le rythme du temps, et en ouvrant la voie à des reconfigurations et à des reclassements par lesquels le cours des choses peut bifurquer. Pour comprendre la réception de l'attentat, il ne fallait pas s'en tenir au cadre trompeur de " réactions " stéréotypées et complaisamment mises en scène, mais chercher à suivre de très près les diverses lectures, les diverses représentations et les divers usages qui «font» autant l'événement et ses effets que le geste même de l'assassin, dont ils diffusent, démultiplient et redessinent l'appréhension initiale.

11 Je me suis arrêté assez longuement sur les hommages, les récits, les images, mais aussi sur les réflexions théoriques qui répondent au choc de l'attentat. En analysant, dans ces réponses, la part des conventions, mais aussi des audaces et des dilemmes, on mesure l'intensité d'un retentissement qui traverse l'histoire sociale mais aussi l'histoire culturelle et intellectuelle du premier XIX ${ }^{e}$ siècle, à l'orée du romantisme. Il s'agissait aussi, bien sûr, selon une perspective politique peut-être plus classique, de s'interroger sur les moyens utilisés pour faire de l'émotion provoquée par la mort du duc de Berry, un point d'appui de la réaction royaliste. Si la mesure de l'adhésion collective à une représentation reste un défi majeur posé à l'analyse historique, divers indices, comme l'épuisement rapide du marché éditorial, le succès de la contre-orchestration libérale ou le peu d'empressement à éterniser dans la pierre le souvenir du 13 février, permettent de souligner au total les contradictions et les lignes de faille qui entravent l'exploitation politique du pathétique. Les effusions consensuelles du mélodrame familial ou conjugal sont rapidement supplantées par la radicalité du clivage systématique qui oppose ultras et libéraux, et le virage à droite de la monarchie s'effectue dans la douleur et l'ambiguïté. Seule la naissance postérieure de l'enfant du miracle paraît pouvoir concrétiser le réel espoir d'un renouveau du sentiment royaliste.

Reste que, avant même l'avènement du ministère ultra, la monarchie a bel et bien pris, au printemps 1820 , un tournant autoritaire dont les sources policières ou judiciaires permettent de mesurer la portée. Il est remarquable que cette réaction soit le fait d'acteurs politiques classés comme modérés, membres de "l'équipe" du second ministère Richelieu, qui se présente comme un recours contre les "extrêmes". L'analyse précise de la reconfiguration politique de 1820 et du passage à droite des hommes du centre, reste largement à mener; elle me paraît une perspective intéressante, qui pourrait s'intégrer dans un effort plus vaste pour relire notre histoire politique et la part qu'y tient un courant modéré prompt à s'accommoder des moyens de gouvernement les plus illibéraux.

13 2) C'était aussi l'intérêt de l'enquête, de permettre un regard plus rapproché sur ces hommes de pouvoir aux prises avec les mirages de la conspiration. Le corpus d'archives judiciaires et policières est riche mais composite et difficile à saisir, aussi bien comme une entité significative que dans ses directions multiples et parfois aléatoires. Là aussi pourtant, un questionnaire en plein renouvellement obligeait à creuser l'analyse. Il fallait bien sûr préciser les soubassements juridiques et institutionnels mais sans 
prendre seulement l'enquête comme dispositif répressif ou comme processus fonctionnel autonome - conduisant d'ailleurs à un relatif échec puisque Louvel paraît seul devant la haute cour les 5 et 6 juin 1820. L'enjeu était plutôt d'en interroger la pratique et les résultats sur le plan intellectuel ou cognitif, pour rattacher autant que possible l'instruction judiciaire au modèle plus large de l'enquête comme mode dominant de production du vrai dans une société démocratique. S'il n'est de science que de ce qui est caché, quelle science la police judiciaire produit-elle en traquant le complot? Une science hybride, certes, combinaison de présupposés puissants, d'un savoir pratique et de résultats empiriques originaux; une science impure donc, fortement biaisée par des impératifs politiques; mais une tentative néanmoins d'élucidation du réel, rendue publique qui plus est par deux bilans imprimés, et d'autant plus digne d'intérêt au total dans une époque travaillée par le désir d'autoreprésentation.

14 Après avoir suivi les logiques d'ensemble qui guidaient la prolifération des pistes et leur résolution, j'ai également essayé d'ouvrir la boîte noire de l'enquête, la boîte noire qu'est l'enquête. Il y a là un travail considérable à mener sur les innombrables dossiers que nous ont laissé magistrats et fonctionnaires du XIX siècle; même dans le seul cadre de l'affaire Louvel, avec plusieurs centaines de dossiers, il était difficile, et sans doute d'ailleurs peu utile, de prétendre à l'exhaustivité. A travers les divers cas étudiés, l'intérêt le plus frappant de ces sources tient à ce qu'on peut appeler l'interactivité de l'enquête, c'est-à-dire la part qu'y jouent, comme dénonciateurs ou comme témoins, ceux qui ne sont pas a priori des acteurs de la police judiciaire. Pour le dire brièvement, ces dossiers donnent à étudier, au niveau le plus fin des relations sociales, la façon dont l'autorité et la domination symbolique se rejouent constamment à travers le processus d'enquête. C'est aussi ce qui permet de comprendre l'importance des affaires les plus dérisoires, et la logique de l'accumulation bureaucratique: dire l'insignifiance ou l'ineptie d'une parole ou d'un bruit, c'est affirmer le pouvoir fondamental de faire la part du vrai et du faux, de l'essentiel et de l'accessoire, et poser la raison, l'anti-rumeur, en garant de l'ordre social et politique.

3) Si elles ne sont certes pas faites pour réhabiliter une expression populaire profondément disqualifiée, ces archives policières et surtout judiciaires ont néanmoins l'effet de conserver, notamment en tant que corps de délit éventuel, les bribes de conversations ordinaires que rien ne devait nous faire parvenir. A ce titre, elles permettent effectivement, après de patients préalables, de compléter le spectre social des attitudes et des discours suscités par l'événement et de retrouver en quelque sorte l'humble sous-sol de l'édifice démocratique. Pour autant, il ne faut pas non plus surestimer le trésor enfoui dans ces archives : la frustration est plus fréquente que la jubilation devant des dossiers souvent elliptiques. Il est là aussi difficile de procéder autrement que par l'étude de certains cas mieux documentés. L'affaire incitait évidemment à se pencher particulièrement sur Louvel, dont le cas m'a très tôt paru aussi exemplaire qu'exceptionnel. Exemplaire : le parcours d'un enfant de la Révolution déboussolé par les revirements politiques successifs des élites; exceptionnel: le passage à l'acte et la mise en lumière consécutive de son comportement et de son discours. Au total, le cas de Louvel invite à s'interroger sur l'articulation du singulier et du collectif dans l'histoire et sur le sens d'une violence politique revendiquée comme une forme d'expression, et répondant à de puissants mécanismes d'exclusion. 
16 A cet égard, Louvel ne manquait pas d'émules dans la France de 1820, marquée par une expression séditieuse et un imaginaire régicide foisonnants, bien au-delà des cercles de la conspiration effective ou vraisemblable. Au-delà même de l'insulte au roi, cette forme triviale de l'adresse, je me suis surtout efforcé de mettre en relief l'intensité de la rumeur et de l'échange social suscités par l'attentat. Il fallait contrecarrer la logique dépréciative des enquêteurs pour retrouver l'autonomie des attitudes et des propos tenus dans le cadre des sociabilités les plus quotidiennes. Insister sur le caractère fondamentalement endogène, ou "normal», de la rumeur comme appropriation populaire de l'événement, et sur le cadre explicatif de la conversation, c'est tenter de dégager les traits de l'homo politicus né de la Révolution, en dépit de la gangue fortement ancrée et persistante des discours et des représentations qui dénient ou censurent jusqu'à son existence même. C'est tenter enfin de retisser le fil de la "politisation" du plus grand nombre, entre les expériences fondamentales de la Révolution et de la guerre et le retour du refoulé démocratique à partir de 1830 .

\section{NOTES}

1. Cette présentation succincte, proche de l'exposé de soutenance, est dépourvue des références bibliographiques.

2. AN CC 503-514; à quoi s'ajoutent notamment des dossiers de la Police générale (F 7), du ministère de la Justice (BB 18), et de la préfecture de police.

\section{INDEX}

Mots-clés : 2005

these 2005 